\title{
Outpatient management of primary spontaneous pneumothorax: a prospective study
}

\author{
Massongo Massongo ${ }^{1,2}$, Sylvie Leroy ${ }^{3}$, Arnaud Scherpereel ${ }^{1,2,4}$, Fabien Vaniet ${ }^{1,5}$, \\ Xavier Dhalluin ${ }^{1,3}$, Bachar Chahine ${ }^{2}$, Céline Sanfiorenzo ${ }^{3}$, Michaël Genin ${ }^{1,6}$ and \\ Charles-Hugo Marquette ${ }^{3}$
}

Affiliations: 'University of Lille Nord de France, Lille, ${ }^{2}$ Pulmonary Dept, Hospital of the University of Lille (CHRU), Lille, ${ }^{3}$ Pulmonary Dept CHU Nice and Inserm U1081, Nice Sophia Antipolis University, Nice, 4 INSERM U1019, Center for Infection and Immunity of Lille (CIIL), Institut Pasteur de Lille, Lille, ${ }^{5}$ Emergency Unit, Valenciennes Hospital, Valenciennes, and 'BBiostatistics Unit, University and Hospital Lille Nord de France, Lille, France.

Correspondence: C-H. Marquette, Service de Pneumologie, Oncologie Thoracique et Soins Intensifs Respiratoires, Université de Nice Sophia Antipolis, Hopital Pasteur, Centre Hospitalier Universitaire de Nice, 30 avenue de la Voie Romaine, 06000 Nice, France. E-mail: marquette.chlachu-nice.fr

ABSTRACT We prospectively assessed the safety and cost saving of a small-bore drain based procedure for outpatient management of first episodes of primary spontaneous pneumothorax.

Patients were managed by observation alone or insertion of an 8.5-F "pig-tail" drain connected to a oneway valve, according to size and clinical tolerance of the pneumothorax. All patients were reassessed after $4 \mathrm{~h}$, on the first working day after discharge and on day 7. Patients still exhibiting air leak on day 4 underwent thoracoscopy. The primary end-point was complete lung re-expansion at day 7 .

60 consecutive patients entered the study. $48(80 \%)$ met the definition of large pneumothorax. The success rate was $83 \%$. The 1 -year recurrence rate was $17 \%$. $36(60 \%)$ patients were discharged after $4 \mathrm{~h}$ and $50 \%$ had full outpatient management. No severe complication was observed. The mean \pm SD length of hospitalisation was $2.3 \pm 3.1$ days. This policy resulted in about a $40 \%$ reduction in hospital stay-related costs.

The present study supports the use of a single system combined with a well-defined management algorithm including safe discharge criteria, as an alternative to manual aspiration or chest tube drainage. This approach participates in healthcare cost-savings.

@ERSpublications

Primary spontaneous pneumothorax can be managed outside the hospital, subject to strict predischarge safety criteria http://ow.ly/qVxcd

For editorial comments see page 334 .

Received: Nov 62012 | Accepted after revision: May 312013 | First published online: June 132013

Support statement: This study was supported by the RESPIR Foundation.

Conflict of interest: None declared.

Copyright @ERS 2014 


\section{Introduction}

The American College of Chest Physicians (ACCP) [1], the Belgian Society of Pneumology (BSP) [2] and the British Thoracic Society (BTS) [3] guidelines recommend the evacuation of air for large primary spontaneous pneumothorax (PSP) and observation alone for the small ones. Evidence keeps growing on the efficiency of observation alone for selected patients with PSP [4], and the idea of abandoning conventional chest tube drainage (CTD) is now considered [5]. Discrepancies remain about the definition of a "large PSP" and the procedures recommended to evacuate the air. While the ACCP recommends the use of a small-bore drain, the BTS and BSP recommend manual aspiration with a needle catheter as a first step, followed by CTD in case of at least one unsuccessful manual aspiration attempt $[1,2,3]$. These new policies aim to manage a benign condition such as PSP in an easy and cost-effective way, while remaining safe.

Manual aspiration, while being less invasive and more comfortable than CTD, has an immediate success rate ranging from $50 \%$ to $68 \%[4,6-8]$. Thus, up to $50 \%$ of patients undergoing manual aspiration will ultimately undergo CTD. Moreover, even in cases of successful manual aspiration, an additional observation period of 3-6 h in the emergency room (ER) is recommended [1]. Therefore, although the quick and effective manual aspiration looks attractive, more than half of patients end up hospitalised with CTD, after a cumulative total of $6 \mathrm{~h}$ procedure and observation time in the ER.

In order to simplify the management of PSP we conducted a study published in 2006 [9] evaluating a serialstepped approach based on a small-bore catheter connected to a one-way valve. This approach was as safe and effective as CTD alone or combined with manual aspiration, with a shorter length of hospital stay $[4,6-8,10,11]$.

The present study was conducted in order to test whether our stepwise approach with a single system [9] also applies to the outpatient management of PSP in real-life conditions.

\section{Methods}

Study design

The study aimed to assess the safety and effectiveness of outpatient management of PSP with a previously described procedure. This was an observational, prospective and noncontrolled study. It was conducted at the Lille University Hospital (Lille, France), from July 2006 to January 2008. Each patient was managed according to our algorithm (fig. 1) and followed for 2 years after the PSP episode.

\section{Patients and selection criteria}

All consecutive patients, aged $\geqslant 16$ years presenting with a first episode of PSP were eligible. To be enrolled, patients had to give an oral informed consent (from a parent or any advocate if aged $<18$ years). Exclusion criteria were as follows: previous ipsilateral episode of PSP, suspected or known underlying pulmonary disease, recent history of trauma, iatrogenic pneumothorax and acute condition on admission.

A detailed information letter was given to each patient before inclusion. The study was approved by the ethical committee of Lille University Hospital.

\section{Procedures and protocol}

Each patient underwent baseline assessment including past history data collection, clinical examination and posteroanterior chest radiography. The pneumothorax was classified as small or large according to the BSP guidelines [2], i.e. a large pneumothorax features lung dehiscence over the whole height of the lateral chest wall. All patients then underwent the following four steps (fig. 1): 1) initial management (pleural drainage or observation); 2) hour 4 clinical and radiological re-evaluation; 3) hospital discharge or hospitalisation in the pulmonary ward; and 4) follow-up. Discharge safety criteria included: 1) patient in stable condition; 2) time to reach the hospital from patient's home $<1$ h by any means of transport; 3 ) patient not living alone; 4) patient able to understand and implement instructions given in case of problem; and 5) time of discharge before 20:00 h. This latter criterion was chosen since, realistically, it seemed unreasonable to discharge a patient at night, within minutes of pneumothorax treatment.

Initial management in the ER

Patients with a small PSP had simple clinical observation, whereas those with a large PSP or breathlessness underwent pleural drainage. The procedure was performed at the bedside, under local anaesthesia, by the attending emergency physician. We used a 8.5-F Fuhrman catheter with a Heimlich valve (C-PPD-850 and C-CASP-A-FORD; Cook Critical Care, Bloomington, IN, USA). The catheter was inserted in the second or third anterior intercostal space on the midclavicular line, the patient lying in a semirecumbent position, following the Seldinger technique. It was then attached to the one-way valve. Folded sterile gauze was set as 


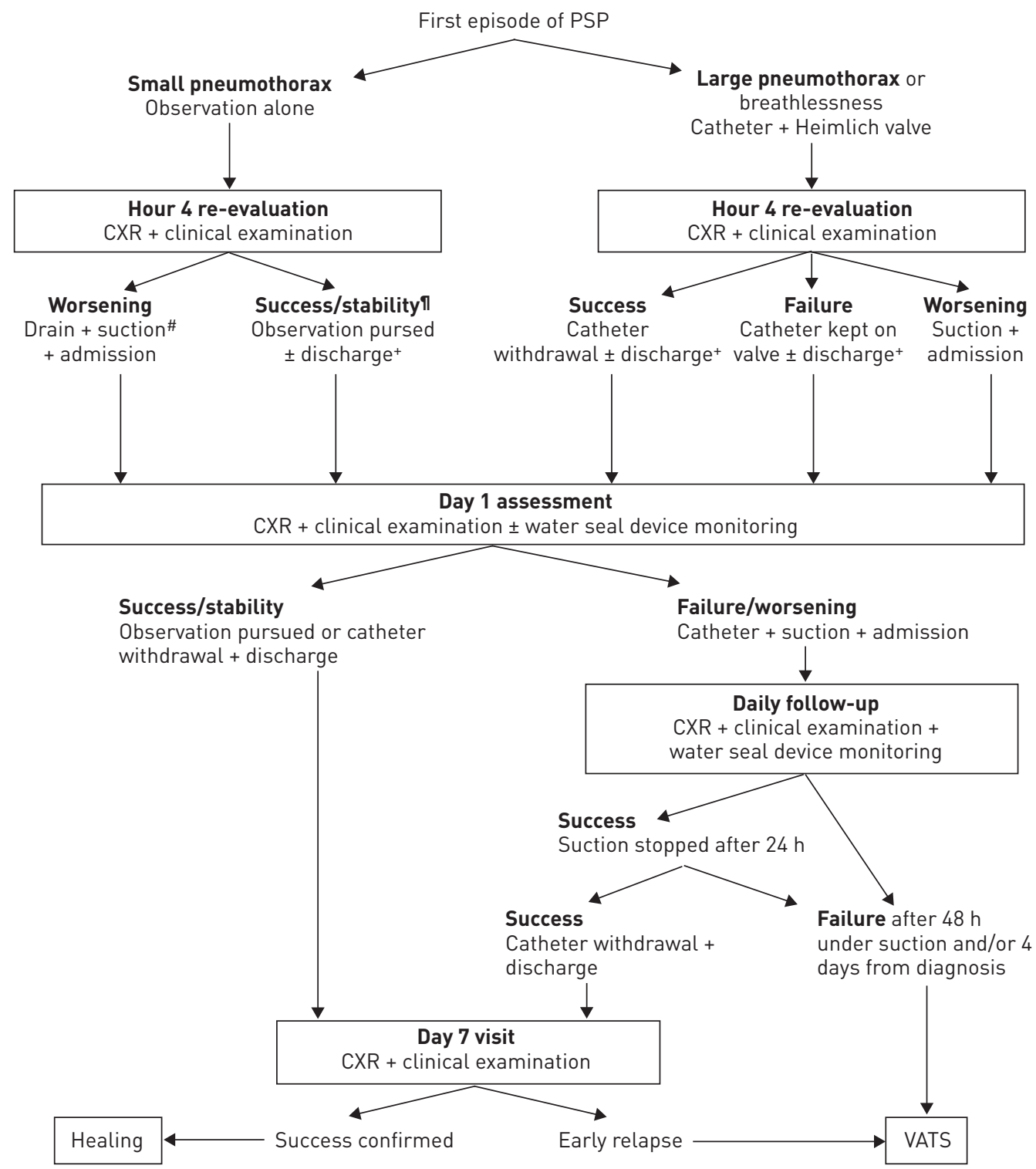

FIGURE 1 Management algorithm of primary spontaneous pneumothorax (PSP). Success: complete or nearly complete lung re-expansion; failure: persisting lung dehiscence on chest radiography or air leak on suction water seal device, without worsening; worsening: increase of pneumothorax size on chest radiograph or clinical deterioration. CXR: chest radiograph; VATS: video-assisted thoracoscopy. ${ }^{\#}$ : suction at $-10--20 \mathrm{cmH}_{2} \mathrm{O}$, could be applied at any time if poor clinical tolerance; ${ }^{\circ}$ : stability applicable for small pneumothorax only; ${ }^{+}$: discharge only if all discharge criteria present.

a pillow under the catheter to avoid kinking (fig. 2). Step-one painkillers were given as needed. Oxygen was administered only for correction of arterial hypoxaemia, if present.

\section{Hour 4 re-evaluation}

Each patient had a clinical evaluation and a posteroanterior chest radiograph. Patients with a small pneumothorax who showed clinical and radiological stability and who fulfilled all five discharge criteria were discharged with an appointment with the pulmonologist in the outpatient clinic on the next working day. Here again, in a realistic approach, we considered the "next working day" as closer to real-world management of a benign condition such as PSP than a strict " $24-\mathrm{h}$ follow-up", which could be in the middle of a weekend when the outpatient clinic is closed. Patients undergoing pleural drainage (large pneumothorax), whose chest radiograph showed complete or almost complete lung re-expansion (i.e. only a very small $<10$-mm rim of apical air) and who fulfilled all five discharge safety criteria had their catheter withdrawn without previous clamping and with no additional chest radiography. They were then discharged with an appointment in the outpatient clinic on the next working day. Those who showed 
FIGURE 2 A Fuhrman pleural drain inserted in the left third intercostal space, attached to a Heimlich oneway valve, for treatment of primary spontaneous pneumothorax. Folded sterile gauze was set as a pillow under the catheter to avoid kinking. An adhesive transparent bandage was applied, while additional adhesive tapes secured the valve on the skin.

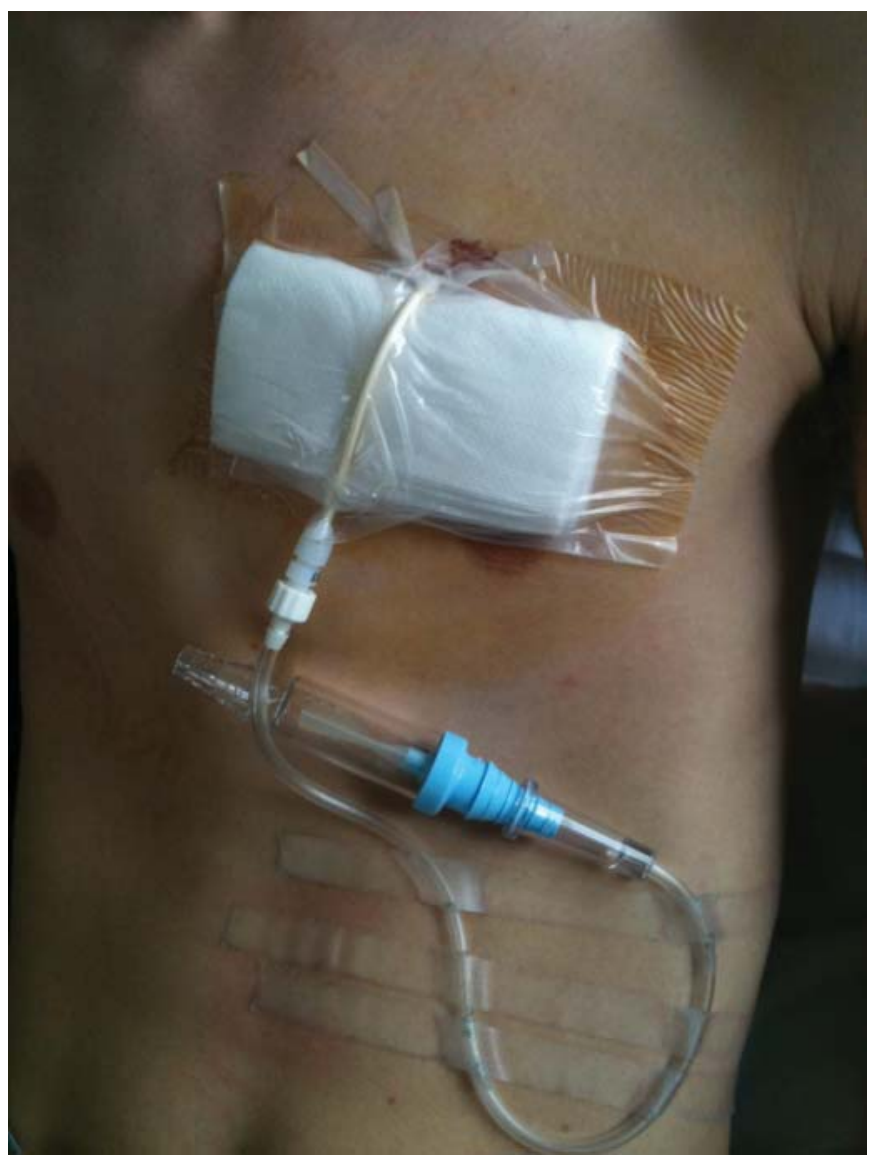

incomplete lung re-expansion and fulfilled all five discharge safety criteria were discharged with their pleural catheter kept on the valve and an appointment in the outpatient clinic on the next working day. Recommendations regarding the use of step-one pain killers were given before discharge. Any patient who did not fit one of the discharge criteria and especially criterion number 5 (time of discharge), stayed in the ER and was re-evaluated the next morning. In patients with a pleural catheter, the catheter was connected to a four-chamber device for suction, in case of acute dyspnoea.

Day 1 re-evaluation (first working day after ER admission)

Patients were clinically and radiologically re-evaluated as at hour 4. For the patients who stayed overnight in the ER this re-evaluation took place there on the morning following their admission. For those who were discharged at hour 4, the day-1 re-evaluation took place in the outpatient clinic, either on the morning following their admission to the ER for patients who were discharged from Sunday to Thursday, or on the next working day for those who were discharged on another day.

Patients who had their catheter in place and showed complete or nearly complete lung re-expansion underwent catheter withdrawal without clamping and additional chest radiography and were given a new appointment on day 7. Those with persisting pneumothorax were admitted to the pulmonary ward and the valve was replaced by a four-chamber water seal device, with a -10- -20-cm $\mathrm{H}_{2} \mathrm{O}$ suction applied for 24-48 h.

Those who had no tube at day 1 and who showed persisting lung re-expansion were given a new appointment on day 7. Those who showed early relapse of their pneumothorax had a new pleural catheter inserted which was connected to a four-chamber water seal device with suction applied for $24-48 \mathrm{~h}$.

After $24-48 \mathrm{~h}$, if the chest radiograph confirmed the lung re-expansion, suction was stopped provided air leak was absent and the drain was kept on water seal device (acting as a one-way valve) for an additional $24 \mathrm{~h}$. It was then withdrawn in case of success. If not, the drainage was maintained under suction, for a maximum of $48 \mathrm{~h}$. Patients with persisting air leak or pneumothorax at day 4 underwent chest computed tomography and were proposed for video-assisted thoracoscopy for definitive treatment. For such patients, the day 7 visit was cancelled and they were considered as failure. 


\section{TABLE 1 Patient characteristics at baseline}

$\begin{array}{lc}\text { Subjects } & 60 \\ \text { Age years } & 25.6 \pm 6.5(16-48) \\ \text { Sex male/female } & 55 / 5 \\ \text { BMI kg. } \text {-2 }^{-2} & 20.6 \pm 2.1(17.3-25.0) \\ \text { Smoking status yes/no } & 48 / 12 \\ \text { Smoking duration median (range) pack-years } & 9(2-30) \\ \text { Comorbidities } & 5 \\ \text { Time to diagnosis" days } & 1.2 \pm 2.9(0.5-21) \\ \text { First symptom } & \\ \quad \text { Pain/dyspnoea } & 49 / 14 \\ \quad \text { Rest/effort } & 48 / 12 \\ \text { Pneumothorax side } & \\ \quad \text { Right } & 31 \\ \quad \text { Left } & 29 \\ \text { Pneumothorax size } & \\ \quad \text { Large } & 48 \\ \quad \text { Small } & 12\end{array}$

Data are presented as $\mathrm{n}$ or mean $\pm \mathrm{SD}$ (range), unless otherwise stated. BMI: body mass index. ${ }^{\#}: 0.5$ day is any delay from 0 to $12 \mathrm{~h}$; ${ }^{\Uparrow}$ : some patients experienced both pain and dyspnoea as first symptoms.

\section{Day 7 visit}

This took place in the outpatient clinic and intended to confirm the recovery. Clinical evaluation and chest radiography were performed. Information and instructions were given to the patient about relapses (symptoms and management) and their prevention.

\section{End-points and statistics}

The primary end-point was the success rate of PSP management at day 7, defined as the percentage of patients with a complete or almost complete persistent lung re-expansion on chest radiograph. Secondary end-points were the percentage of subjects with full outpatient management; the mean length of hospital stay; the mean cost of management (mean length of hospitalisation stay multiplied by the 1-day hospitalisation fees for a medical unit in a French public hospital); the 2-year actuarial recurrence rate (defined as the proportion of patients presenting with a first ipsilateral relapse within the 2 years following the first episode); side-effects; and complications of medical procedures. Qualitative data are provided as percentages of the population and demographics and other quantitative data as mean or median \pm SD. Time-dependent data were expressed using the Kaplan-Meier method. Wilcoxon and Fisher statistical tests were used to analyse the quantitative data and qualitative values, respectively. $\mathrm{p}<0.05$ was deemed statistically significant.

\section{Results}

Patients

60 consecutive patients entered the study and all were followed for $\geqslant 2$ years. The main characteristics of patients and pneumothoraces are displayed in table $1.80 \%$ were smokers, $91 \%$ were males and the body mass index was $<21.5 \mathrm{~kg} \cdot \mathrm{m}^{-2}$ for $>50 \%$ of subjects. $48(80 \%)$ patients met the definition of large pneumothorax and $12(20 \%)$ met the definition of small pneumothorax. Among patients with a large pneumothorax, only two had a $<2-\mathrm{cm}$ rim of air between the lung margin and the chest wall at the level of the hilum. Thus, $96 \%$ of patients classified as having a large pneumothorax satisfy the BTS criteria for large pneumothorax [3]. Small and large PSP did not differ in terms of age or cigarette consumption (data not shown).

\section{Hour 4 re-evaluation}

All patients with a small PSP $(n=12)$ were treated conservatively (none of them complained of breathlessness) and were discharged at hour 4 without further complication (figs 3 and 4). They had a persistent complete or almost complete lung re-expansion at day 7.

All patients with a large PSP $(n=48)$ had pleural drainage with the 8.5 -F pig-tail pleural catheter connected to the one-way valve. More than 25 different practitioners inserted the pleural catheters, only five of them being respiratory physicians. Nine patients showed lung re-expansion on hour 4 re-evaluation and were discharged after having their catheter withdrawn. 15 were discharged with their pleural catheter kept on 
FIGURE 3 Flow diagram for progress of patients through management and outcome. PSP: primary spontaneous pneumothorax

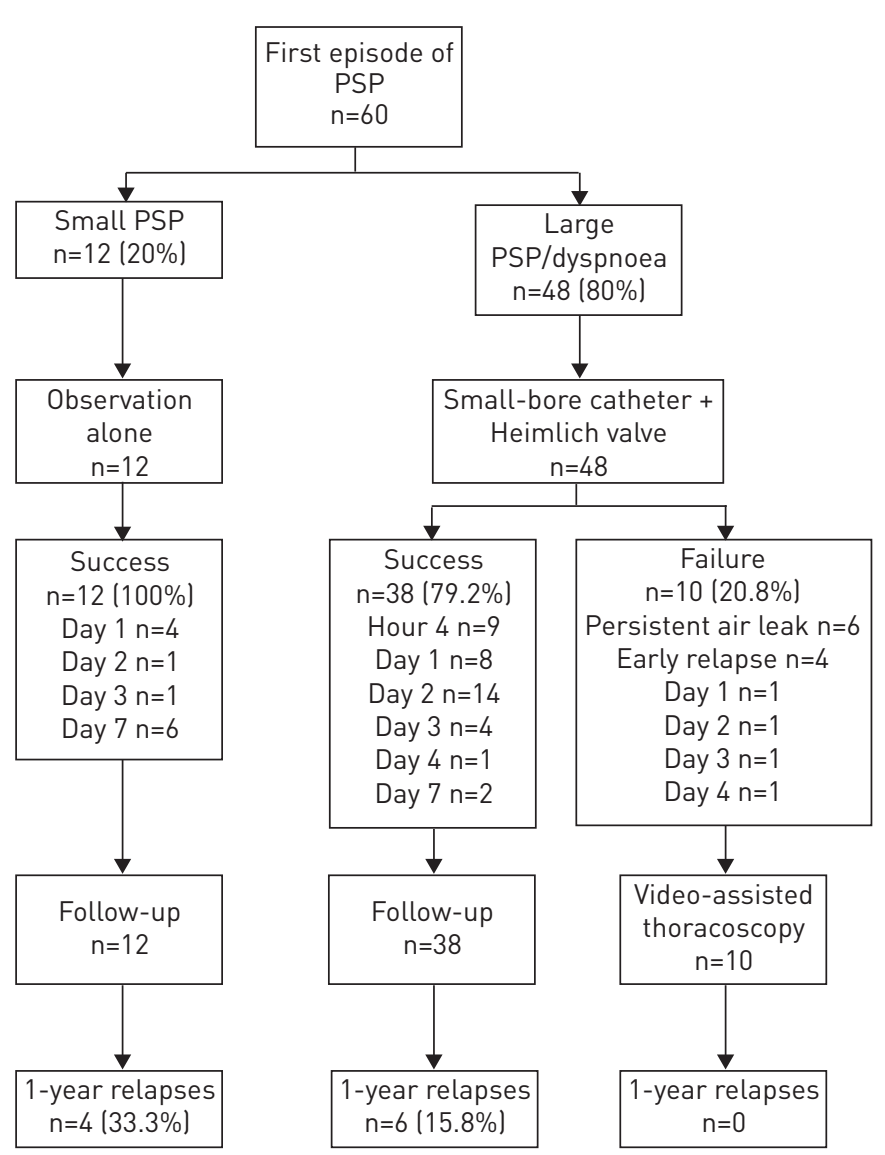

valve since showing incomplete lung re-expansion and fulfilling all five discharge safety criteria. Two patients with a large pneumothorax required suction applied to the pleural catheter within the first $4 \mathrm{~h}$ of observation because of acute dyspnoea and increase in pneumothorax size. 22 additional patients with a large PSP did not fulfil the five discharge criteria and stayed overnight. Thus, 36 (12 small PSP and 24 large PSP) out of the 60 patients $(60 \%)$ were discharged early (hour 4 ).

\section{Day 1 (first working day after ER admission) to day 7 re-evaluation}

Among the 36 early discharged patients six were readmitted 1 day $(n=4), 2$ days $(n=1)$ and 3 days $(n=1)$ after discharge as the first re-evaluation as outpatients showed insufficient lung re-expansion or very early relapse (figs 3 and 4). None of them presented acutely when reassessed at day 1 . These patients had their pleural catheter placed under suction as per protocol.

Among the 24 patients staying overnight, 14 required additional suction, as per protocol, because their day 1 re-evaluation did not show lung re-expansion. 20 could eventually be discharged after having their pleural catheter withdrawn at day $1(n=8)$, day $2(n=2)$, day $3(n=7)$ and day $4(n=3)$. Step-one pain killers were sufficient in all but six patients who had their pleural catheter placed under suction and needed step-two analgesics. Only two patients whose pleural catheters were under suction required supplemental oxygen because of transient hypoxaemia.

\section{Primary end-point}

50 patients (12 small PSP and 38 large PSP) fulfilled the definition of success ( $83 \%$ success rate). 10 (large PSP) failed to re-expand their lung or had air leak $>4$ days after ER admission and thus underwent thoracoscopy. No significant clinical or radiological difference was observed between success and failure groups (table 2).

\section{Secondary end-points}

Two patients developed a procedure-related complication (table 3). One presented an a vacuo re-expansion pulmonary oedema $2 \mathrm{~h}$ after suction was applied to his drain. This complication resolved spontaneously 


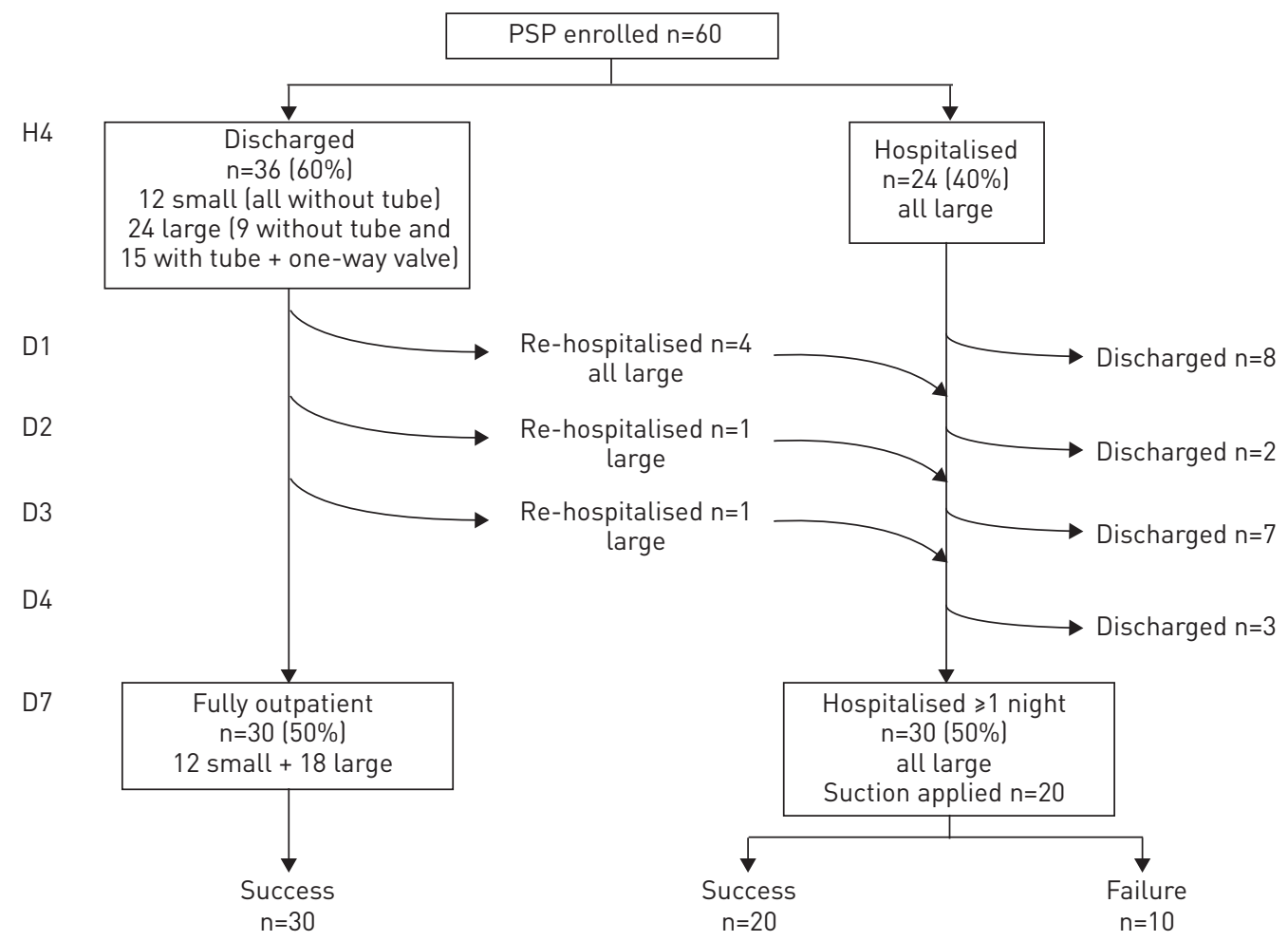

FIGURE 4 Flow diagram representing patient admissions and discharges during the study period. PSP: primary spontaneous pneumothorax; H4: hour 4; D1-D7: day 1-day 7.

and the patient was discharged at day 3. One showed a small pleural effusion at day 7 follow-up, 4 days after drain withdrawal, and had $800 \mathrm{~mL}$ of blood-tinged fluid evacuated in the outpatient clinic.

$30(50 \%)$ patients were fully managed as outpatients (12 small PSP and 18 large PSP); six needed to be readmitted, and 24 had a single-course hospital stay. The mean length of hospital stay was $2.3 \pm 2.5$ days (range $4 \mathrm{~h}$ to 12 days). The mean drainage duration was $2.3 \pm 3.1$ days (range $0-12$ days). 20 patients needed suction, including the 10 who finally underwent a thoracoscopy. Thus, two-thirds of the 60 patients did not need any connection to wall suction, and could walk around during the whole time course of their pneumothorax management.

Within the first year, an ipsilateral relapse occurred in four out of 12 patients with a small PSP (at 1, 4, 5 and 9 months) and six out of 48 patients with a large PSP ( 3 weeks $n=1 ; 1$ month $n=1 ; 1.5$ months $n=2$; 3 months $\mathrm{n}=1$; and 12 months $\mathrm{n}=1$ ) after discharge. The 2 -year recurrence rate was $16.7 \%$. Relapses appeared to be significantly associated with young age $(\mathrm{p}=0.015)$, with eight out of 10 occurring among

TABLE 2 Comparison of patients: success of treatment group versus failure of treatment group

\begin{tabular}{lccc} 
& Success & Failure & p-value \\
\hline Subjects & 50 & 10 & \\
Age years & $25.0 \pm 6.6$ & $28.8 \pm 4.9$ & 0.19 \\
Sex male/female & $45 / 5$ & $10 / 0$ & Not valid \\
Smokers \% & 80.8 & 80.0 & 0.22 \\
Pack-years in smokers & $7.4 \pm 7.0$ & $8.5 \pm 5.1$ & $>0.50$ \\
First symptom pain/dyspnoea & $41 / 12$ & $8 / 2$ & 0.41 \\
First symptom rest/effort & $39 / 8$ & $7 / 1$ & Not valid \\
Time to diagnosis days & $1.3 \pm 3.2$ & $0.6 \pm 0.3$ & $>0.30$ \\
Size of pneumothorax small/large & $12 / 38$ & $0 / 10$ & 0.18 \\
Side of pneumothorax left/right & $24 / 26$ & $5 / 5$ & 1.0 \\
\hline
\end{tabular}

Data are expressed as $\mathrm{n}$ or mean $\pm \mathrm{SD}$, unless otherwise stated. 
TABLE 3 Summary of the results for the study end-points

Success rate at day 7

Part or full outpatient management

Mean length of hospitalisation days

$30(50)$

Mean cost of management per episode $€$

$2.3 \pm 2.5$

2-year recurrence rate

2710.36

Complication rate

$10(16.7)$

2 (3.3)

Data are presented as $\mathrm{n}(\%)$ or mean \pm SD.

patients aged $<25$ years, and small PSP ( $\mathrm{p}=0.04$ ), with four out of 12 in patients with small PSP versus six out of 48 patients with large PSP experiencing a relapse.

The cost of the device is $€ 107$ ( $€ 65$ for the Fuhrman drain and $€ 42$ for the Heimlich valve). Taking into consideration hospitalisation fees in a pulmonary department (€1194 per day), the mean cost of management was $€ 2710$ per patient.

\section{Discussion}

In this study, conducted under real-life conditions, nearly two-thirds of patients suffering a first episode of PSP could be discharged after $4 \mathrm{~h}$ in the ER. Management of pneumothorax needed wall suction in only one-third of patients. The short-term success rate was remarkably high (83\%) and the 2-year actuarial recurrence rate was low $(16.7 \%)$.

Demographic characteristics of our study population agree with common findings in PSP, confirming that this disease usually occurs at rest in young, tall, thin smoking males [1,6-9, 12--14]. The 20\% proportion of small PSP is close to our previous findings [9] and is less than that reported in the literature. This might be related to differences in defining the size of a pneumothorax. ACCP guidelines consider a pneumothorax as large when the distance from apex to cupola is $\geqslant 3 \mathrm{~cm}$ [1], whatever the dehiscence on the axillary line. In contrast, BTS guidelines consider a pneumothorax as large when there is a $\geqslant 2 \mathrm{~cm}$ rim of air between the lung margin and the chest wall at the level of the hilum $[3,12]$. In the present study, $96 \%$ of the patients classified as having a large pneumothorax satisfied the BTS criteria. Interestingly, none of our patients classified as having a small pneumothorax required drainage because of breathlessness. Our 1-week success rate is close to the success rates reported in the literature with standard chest tubes [6-8, 11] and with the $85 \%$ success rate that we previously reported with a similar technical approach but with a $100 \%$ hospitalisation policy [9]. BTS and BSP guidelines recommend manual aspiration as a first step for patients with a large PSP, followed by CTD in case of at least one unsuccessful manual aspiration attempt $[2,12]$. This allows $\sim 50 \%$ of the patients with a large pneumothorax to be discharged after manual aspiration followed by 3-6 h observation in the ER. We previously showed that, in patients with a first episode of pneumothorax, provided a well-defined serial steps approach is used, the single small catheter/Heimlich valve system could safely replace first-step manual aspiration but also subsequent CTD, avoiding prolonged ER observation, subsequent hospitalisation and unnecessary additional discomfort related to secondary tube thoracostomy in those patients who fail manual aspiration [9]. As previously shown for manual aspiration [6] the size of the pneumothorax on admission did not influence the success or the failure of our single small catheter/Heimlich valve approach.

Interestingly we could confirm that, provided strict discharge safety criteria are applied, portable smallcalibre catheter and Heimlich valve can be used in ambulatory care management [15].

In terms of quality of life, this approach, which allowed full ambulatory management of $50 \%$ of the patients, can be considered as an improvement when compared to the more conservative CTD approach, which would have led to up to $80 \%$ of the patients in a hospital ward, with their chest connected to a tube and a vacuum bottle. Reduction of length of hospital stay is also an important consideration from a health costs point of view. If the average usual 4-day hospital stay of a patient undergoing CTD for a pneumothorax $[6,8]$ is taken into account, the cost saving of the hour 4 discharge in the 24 patients with a large pneumothorax was estimated at $>€ 114000$, even though six of them required a short readmission. If only the patients who are usually hospitalised (large pneumothoraces) are considered, avoiding hospital stay in 18 patients (fully managed as outpatients) out of a total of 48 patients makes a $38 \%$ reduction in hospital stay-related costs.

PSP is a common problem affecting young and healthy people. Given the age and sex-adjusted incidence of PSP $[16,17]$, healthcare facilities serving a catchment population of 1 million inhabitants are expected to 
treat 125 cases of PSP every year [18, 19]. In terms of quality of life and health costs saving, all efforts must be made ensure ambulatory care management of these young and otherwise healthy people. Manual aspiration is recommended as a first step by several guidelines [2, 12] but conservatism of doctors and drawbacks inherent to the manual aspiration technique hinder the wide implementation of the technique. The present study supports the use of a single system (portable small-bore catheter connected to a one-way valve) combined with a well-defined management algorithm including appropriate discharge safety criteria, as an alternative to manual aspiration. Despite significant limitations (small sample size, lack of a randomised controlled intervention trial, single centre experience and applicable only to patients living near to the hospital) this study should prompt further improvement in management of pneumothorax, including controlled prospective studies comparing manual aspiration, the gold standard approach, to catheter plus valve plus immediate "back home" in patients with large pneumothoraces.

\section{References}

1 Baumann MH, Strange C, Heffner JE, et al. Management of spontaneous pneumothorax: an American College of Chest Physicians Delphi consensus statement. Chest 2001; 119: 590-602.

2 De Leyn P, Lismonde M, Ninane V, et al. Guidelines Belgian Society of Pneumology. Guidelines on the management of spontaneous pneumothorax. Acta Chir Belg 2005; 105: 265-267.

3 MacDuff A, Arnold A, Harvey J. Management of spontaneous pneumothorax: British Thoracic Society Pleural Disease Guideline 2010. Thorax 2010; 65: Suppl. 2, ii18-ii31.

4 Kelly AM, Kerr D, Clooney M. Outcomes of emergency department patients treated for primary spontaneous pneumothorax. Chest 2008; 134: 1033-1036.

5 Janssen J, Cardillo G. Primary spontaneous pneumothorax: towards outpatient treatment and abandoning chest tube drainage. Respiration 2011; 82: 201-203.

6 Noppen M, Alexander P, Driesen P, et al. Manual aspiration versus chest tube drainage in first episodes of primary spontaneous pneumothorax: a multicenter, prospective, randomized pilot study. Am J Respir Crit Care Med 2002; 165: $1240-1244$.

7 Andrivet P, Diedaini K, Teboul JL, et al. Spontaneous pneumothorax. Comparison of thoracic drainage vs immediate or delayed needle aspiration. Chest 1995; 108: 335-339.

8 Ayed AK, Chandrasekaran C, Sukumar M. Aspiration versus tube drainage in primary spontaneous pneumothorax: a randomised study. Eur Respir J 2006; 27: 477-482.

9 Marquette CH, Marx A, Leroy S, et al. Simplified stepwise management of primary spontaneous pneumothorax: a pilot study. Eur Respir J 2006; 27: 470-476.

10 Lodi R, Stefani A. A new portable chest drainage device. Ann Thorac Surg 2000; 69: 998-1001.

11 Harvey J, Prescott RJ. Simple aspiration versus intercostal tube drainage for spontaneous pneumothorax in patients with normal lungs. BMJ 1994; 309: 1338-1339.

12 Henry M, Arnold T, Harvey J. BTS guidelines for the management of spontaneous pneumothorax. Thorax 2003; 58: Suppl. 2, ii39-ii52.

13 Waller DA, Forty J, Morritt GN. Video-assisted thoracoscopic surgery versus thoracotomy for spontaneous pneumothorax. Ann Thorac Surg 1994; 58: 372-376.

14 Weissberg D, Refaely Y. Pneumothorax: experience with 1,199 patients. Chest 2000; 117: 1279-1285.

15 Choi SH, Lee SW, Hong YS, et al. Can spontaneous pneumothorax patients be treated by ambulatory care management? Eur J Cardiothorac Surg 2007; 31: 491-495.

16 Melton LJ 3rd, Hepper NG, Offord KP. Incidence of spontaneous pneumothorax in Olmsted County, Minnesota: 1950 to 1974. Am Rev Respir Dis 1979; 120: 1379-1382.

17 Bense L, Eklund G, Wiman LG. Smoking and the increased risk of contracting spontaneous pneumothorax. Chest 1987; 92: 1009-1012.

18 Miller AC, Harvey JE. Guidelines for the management of spontaneous pneumothorax. Standards of Care Committee, British Thoracic Society. BMJ 1993; 307: 114-116.

19 Gupta D, Hansell A, Nichols T, et al. Epidemiology of pneumothorax in England. Thorax 2000; 55: 666-671. 